\title{
Strengthening healthcare providers' capacity for intra-uterine device and contraceptive implant services in humanitarian and fragile settings: a case study from Nepal using the clinical outreach refresher training model (S-CORT)
}

Kamille Gardner

CARE USA

Shabana Zaeem

CARE USA

Jagadishwor Ghimire

IPAS

AKM Anisuzzaman

CARE Bangladesh

Bergson Kakule Syaivuya

CARE

Alison Greer

Women's Refugee Commission

Anushka Kalyanpur

CARE USA

Nguyen Toan Tran ( $\nabla$ tntoan18@gmail.com)

University Of Technology Sydney https://orcid.org/0000-0001-7134-7878

\section{Research}

Keywords: Long-acting and reversible contraceptives, contraceptive implants, intra-uterine devices, capacity development, refresher training, human resources for health, humanitarian settings, preparedness, sexual and reproductive health and rights

Posted Date: February 5th, 2021

DOl: https://doi.org/10.21203/rs.3.rs-183559/v1

License: (a) (1) This work is licensed under a Creative Commons Attribution 4.0 International License. Read Full License 


\section{Abstract}

Background - Access to modern family planning methods, including long-acting and reversible contraceptives (LARCs), to prevent unplanned pregnancy is critical to avert maternal deaths in humanitarian and fragile settings, where clinicians often have limited LARC competencies. This paper explores the perspectives and recommendations of providers and trainers involved in piloting the Clinical Outreach Refresher Training for Sexual and Reproductive Health module (S-CORT) on LARCs in Nepal.

Methods - Qualitative data from end-of-training evaluations, which were self-filled by 15 trainees, a focus group discussion involving 11 of them, and written feedback from five co-trainers were transcribed, coded, and thematically analyzed. Results were intersected with those from pilots held in Bangladesh and the Democratic Republic of Congo.

Results - Results suggest that the module could increase participants' counseling and clinical skills, help anchor readiness for family planning and LARC provision before humanitarian emergencies, and reinforce informed consent, service privacy and confidentiality, and access for underserved populations, including adolescents. Recommendations for improvement included the advanced provision of the learning resources for self-study to promote blended learning and avail more clinical practice time.

Conclusions - When the lack of skilled human resources is a barrier to LARC services in humanitarian and fragile settings, the S-CORT strategy could offer a rapid hands-on refresher training opportunity for clinicians requiring knowledge and skills update. Such a capacity-development approach could be valuable not only for emergency response but also in contexts prioritizing disaster preparedness planning.

\section{Background}

An estimated 218 million women of reproductive age, mostly in low and middle-income countries, want to delay or prevent pregnancy but are not using a modern family planning method despite increased contraceptive use in the past decades [1]. Meeting the unmet need for contraception in these countries and offering all pregnant women and neonates the standard care recommended by the World Health Organization could dramatically reduce unintended pregnancies by $68 \%$, unsafe abortions by $72 \%$, maternal deaths by $62 \%$, and neonatal deaths by $69 \%[2,3]$. Furthermore, approximately two-thirds of maternal deaths worldwide occur in countries affected by fragility and crises [4]. These deaths disproportionately affect women and girls who, in times of crisis, often face multiple sexual and reproductive health (SRH) risks and additional barriers to accessing lifesaving healthcare services, including voluntary contraception [5].

Despite the evidence on the benefits of family planning in fragile and humanitarian settings, several evaluations and reviews have called for special attention on this issue as they found inconsistent to nonexistent service delivery of contraception. Such gaps concern in particular long-acting reversible contraceptive (LARC) methods, which women have been shown to demand and use even in crisis 
contexts, including as part of post-abortion care services [6-10]. Likewise, these services were underrepresented in humanitarian appeals and funding allocations [11], and the topic received limited attention in terms of health intervention research in humanitarian crises [12]. The situation is particularly concerning given the evidence pointing to the importance of a broad range of contraceptive methods for quality contraceptive programming to be responsive to the individual and changing needs of women and girls [12].

As such, the Minimal Initial Service Package (MISP) for SRH in humanitarian settings-an international standard in humanitarian response to be delivered from the onset of a crisis [13]-has strengthened its guidance on contraceptive services over time [14]. The 2010 Edition of the Inter-Agency Field Manual for Reproductive Health in Humanitarian Settings recommended contraceptive provision in rape survivor management and the prevention of maternal deaths and disabilities. It referenced family planning programming as an add-on intervention. In contrast, the 2018 Edition acknowledged the critical issue of unintended pregnancy for women and adolescent girls in crisis settings and repositioned voluntary contraception as a standalone MISP objective [15]. Furthermore, the revised MISP has also listed contraception as a core component of post-abortion and safe abortion care services [14].

\section{LARCs in humanitarian settings}

Recent humanitarian case studies in Bangladesh (Cox's Bazar), Nigeria (Borno State), and Mozambique (areas affected by Cyclone Idai) have shown some progress regarding the availability of contraceptive services except for LARCs and identified the limited capacity of health providers as a significant impediment [16-18]. Additionally, providers required enhanced mentorship and supervision so that their provision of LARC services could better align with client-centered and rights-based principles, such as confidentiality and informed consent. Therefore, to help avail clinical staff with the necessary knowledge and skills to provide these services in crisis-affected and fragile settings, the Training Partnership Initiative of the Inter-Agency Working Group on Reproductive Health in Crisis Settings developed a refresher training course on LARCs. The course adopted an established capacity development strategy known as the SRH Clinical Outreach Refresher Training (S-CORT) [19]. Compared to skill-building courses on similar topics that last a week or more, the accelerated S-CORT model aims to reach out to frontline health providers working in humanitarian contexts, such as nurses and midwives, and refresh their knowledge and skills on lifesaving SRH skills, which they had previously learned but may not have kept up to date. Such training courses last over two to three days, depending on the topic, and usually do not include a clinical practicum with real clients, as trainees are required to have prior clinical experience with clients on the topic to be refreshed. The S-CORT on LARCs was designed in 2018 as a three-day in-person workshop and piloted in 2019 in the fragile and humanitarian contexts of Nepal, Bangladesh, and the Democratic Republic of Congo (DRC). This paper aims to describe the pilot experience in Nepal, including stakeholders' perspectives and recommendations for improvement.

\section{Pilot country}


Nepal has a propensity for natural disasters, such as flash flooding, earthquakes, and landslides. In April 2015, a 7.8-magnitude earthquake impacted nearly one-third of the total population, causing approximately 9,000 deaths and 22,000 injuries and damaging around 900 health facilities [20]. In August 2017, floods and landslides severely affected the lives of 390,000 families across 32 districts [21]. Despite this proclivity to natural disasters, Nepal has made strides in the health sector, reducing its maternal mortality ratio from 553 to 186 per 100,000 live births between 2000 and 2016 [22]. Furthermore, the modern contraceptive prevalence rate among women of reproductive age increased from 26-43\% between 1996 and 2016 [23]. However, a health facility survey conducted by the Ministry of Health found, with regard to contraceptive services, that the overall environment for counseling was substandard with limited privacy and confidentiality [24]. Additionally, only around one in five facilities offered intra-uterine devices and implants, and just over one in ten providers ever received in-service training on LARCs [24].

\section{Methods}

\section{Intervention}

The S-CORT approach supports group-based or individual training with an eye on skills practice and participatory learning. The LARC training package contains a facilitator's guide (see www.iawg.net) and slide sets for the trainer and handouts, checklists, and job aids for participants. Training methodologies include slide-aided interactive presentations, group discussions, questions and answers, role-plays, small group work, case studies, videos, and demonstration and hands-on skills practice with anatomical models along with checklists to guide practice. The training module contents were adapted from the most recent resource materials, including guidance from the World Health Organization, and were adapted for humanitarian contexts $[25,26]$.

\section{Selection of implementing partners}

In 2019, the Training Partnership Initiative of the Inter-Agency Working Group for Reproductive Health in Crises partnered with CARE International, an implementing non-governmental organization solicited through an open application process to pilot the module. Criteria for implementing organizations included having family planning programs within the institutional workplan; prior experience organizing trainings in crisis-affected settings; commitment to supporting capacity development efforts for national and international SRH providers; and capacity to undertake an evaluation of the module and training workshop. CARE, in collaboration with the Family Planning Association of Nepal and the Ministry of Health's National Health Training Center, implemented pilots in Kathmandu, Nepal, in the context of emergency preparedness given the high risk of natural disasters. Similar pilots took place in other contexts: the protracted humanitarian setting of North Kivu province in DRC and the relatively more recent Rohingya refugee camp situation in Cox's Bazar, Bangladesh. Despite differences between the three countries in the types of crisis settings, a common thread was the need for the increased availability of 
providers competent in quality LARC services to help realize a broader mix and choice of contraceptive methods.

\section{Evaluation objectives}

The pilot workshop evaluation had two primary objectives: first, to evaluate the training materials themselves, including the clinical outreach training model, which would help inform the finalization of the design and contents of the learning resource package, and, second, to evaluate the implementation of the training, which would strengthen guidance around organizing such trainings.

The evaluation of the module adopted a mixed-method approach, which included a self-filled pre-test and post-test questionnaire, a competency checklist used for pre- and post-training skills assessment, a selffilled end-of-training evaluation, and qualitative interviews with participants. This case study reports results from Nepal as the collected data were the most complete and could reliably depict the implementation process as compared to data from Bangladesh and DRC, which were not or only partly collected due to time and staff constraints. The results concentrate on the qualitative data of the end-oftraining evaluation and qualitative interviews as these offered the most relevant sources of insights and lessons to improve future implementation.

\section{Data collection}

At the end of the workshop, an independent evaluation staff from CARE conducted a focus group discussion using a semi-structured interview guide. The evaluation teams used convenience sampling for participant selection by inviting trainees to participate. The discussion was audiotaped after obtaining agreement from participants. Research assistants transcribed the recording into English with accuracy checks done by comparing transcripts with the audio file. The focus group discussion gathered 11 out of 15 trainees (all women). Additionally, written comments from end-of-workshop evaluations from all trainees and feedback from five local trainers provided further sources of qualitative data. The data was single-coded and thematically analyzed using QSR NVivo 12 software, a qualitative research management tool.

\section{Ethics and informed consent}

The evaluation tools were part of planned program monitoring, which was not meant to develop and contribute to generalizable knowledge and, therefore, did not form research and necessitate ethical approval [27]-this was verified through our submission to the Western Institutional Review Board (No. 2633824-44635729). The evaluation was considered minimal risk, and workshop participants did not have to complete the evaluation as a condition of workshop attendance nor were they incentivized to participate in the evaluation. Therefore, informed consent was not necessary. Evaluators briefed participants about the voluntary nature of their involvement in the evaluation, the anonymity of all their feedback, and the confidentiality surrounding data management and analysis. There were no patients involved in the evaluation. 


\section{Results}

In February 2019, implementing partners in Nepal ran a three-day training workshop for the all-women team of 15 participants. These were service providers, including eight nurses, six midwives, and one physician. The workshop was led by an all-women team of clinicians comprised of one international lead facilitator and five local co-facilitators who were inducted beforehand to the learning resource packagethese co-facilitators will help carry out future cascade trainings in other districts.

\section{Skills confidence}

Overall, participants found the S-CORT on LARCs effective in refreshing their knowledge and skills. They reported a boost in their confidence in prioritizing family planning and LARC services even in humanitarian settings, stressing the importance of clinical readiness for such interventions.

Earlier, we provided services, but now we are also more focused on how to best provide services in emergency settings. Trainee

Many commented that it was their first exposure to the MISP and underlined the importance of offering $\mathrm{SRH}$ services even in humanitarian settings.

I feel very confident that I can provide the best service for my patients regardless of any disastrous situations. Trainee

Providers agreed on the effect of the training on how they would offer services, stressing the improvement of counseling as a distinct clinical take-away.

Before, balanced counseling was lacking, but after the training, I have started paying close attention to counseling, along with providing other medical services. I have started realizing the importance of counseling the patients before and after providing contraceptives like LARCs. Trainee

\section{Rights and access}

The training appears to have contributed to increasing the awareness and practice of rights-based service provision, such as systematically obtaining informed consent from clients. Ensuring the privacy and confidentiality of services was also a highlight.

I think one thing I would like to say about this training is that it has helped us enhance our performance as it has stressed the idea of patient privacy and confidentiality. Trainee

In their reflection about the geographical barriers inherent to the Nepalese terrain, especially in rural areas, providers understood the importance of expanding choice and ensuring access to LARCs for individuals displaced by crises. 
Sometimes women have to walk five to seven hours to the campsites to receive contraceptives in rural areas of Nepal. So, it is even more important to provide them with LARC methods in order to minimize [the risk of] unwanted and unintended pregnancies. Trainee

Additionally, participants appeared to understand the need for adolescents to have equal access to a wide choice of contraceptives, including LARCs. In this respect, they underlined the need to appropriately counsel adolescents about LARCs, such as recommending a barrier method when needed to prevent sexually transmitted infections.

\section{Training approach}

Overall, trainees and local trainers found the learning resource package user-friendly and balanced between interactive theory and practice.

The package is well organized, has handouts, checklists, is color-coded, and also has demonstration and practice sessions... Everything is readymade here. So easy for us, the right balance of materials. Trainer

Most participants and co-trainers recommended increasing the training to five days with a dedicated day for clinical practice. Instead of returning home every day after the training hours, a residential workshop would be a preferred option with the advantage of allowing more time for group learning and sharing.

If we are able to provide all the materials and equipment in a hotel setting and maybe if we are able to spend the night there, then we would not have to rush back home or rush back in the next morning. We would feel rather relaxed and also would be able to share experiences and learnings after the training session is over among all the participants. Trainee

However, several participants suggested that sharing a hardcopy of the learning materials in advance for self-study could be more time-efficient and allow further opportunities for clinical practice. Such an approach could help successfully keep the workshop to a three-day duration.

I think if we are given the materials in advance, then we can have a look at them and then further discuss it during the session for more efficiency and effectiveness. Trainee

Overall, there was agreement on the advantages of the training localization driven by the S-CORT outreach strategy.

On-the-job training is better. Better if we go there and do it in different districts. Then, participants don't have to travel a long distance. Trainer

\section{Discussion}

The pilots of the newly developed S-CORT curriculum on LARCs took place in fragile or humanitarian contexts in Nepal, Bangladesh, and the DRC. Qualitative results from Nepal suggest that the module helped increase participants' counseling and clinical skills, readiness for family planning and LARC 
provision during humanitarian emergencies, and quality of care, including informed consent, privacy, confidentiality, and access for underserved populations, such as adolescents. Trainees and trainers valued the S-CORT package and training approach but recommended the advanced provision of the materials for self-study to help with the efficiency of the training and effectively keep its duration to three days.

The qualitative results mirrored the end-of-workshop evaluations in both Nepal and DRC: all trainees reported feeling confident inserting and removing intra-uterine devices and implants. Additionally, a vast majority in both countries (26 out of 27 ) were positively impressed by the overall workshop, including the quality of training resources and methodology and the efficient use of time at hand. The qualitative data and knowledge pre-tests and post-tests from the pilot countries also converged with the Nepali findings. In terms of knowledge enhancement, scores increased from $64-91 \%$ in DRC and from $64-93 \%$ in Nepal. As another example, participants from Bangladesh highlighted how the workshop enhanced their knowledge as well as counseling and technical skills. They also recommended a residential workshop but for another reason: to minimize safety threats as they had to "hurry back and forth during sunlight" daily.

Results from the LARC pilot intersect with the findings from previous research on the S-CORT model, which indicated that the strategy respects human rights and quality of care principles on top of being potentially effective in strengthening the knowledge and skills of existing trained service providers and changing their attitudes, such as on safe abortion and post-abortion care $[19,28]$. However, it is helpful to remember the capacity development strategy initially proposed in the S-CORT: a fast on-the-ground training during the acute or post-acute phase of a crisis to refresh the knowledge and skills of providers on SRH lifesaving interventions, which they once studied. In Nepal, trainees and co-trainers acknowledged the potential role of the S-CORT in the context of primary and SRH care and disaster preparedness. The earthquake in 2015 exposed significant preparedness gaps and post-earthquake response in the health sector, including the SRH and family planning areas, as exemplified by the need for increased human resource capacity and readily available supplies [29-31]. Therefore, program managers and policymakers could consider the S-CORT model to refresh providers' capacity in contexts where emergency preparedness efforts are a priority. Further research is necessary to study the barriers and catalysts specific to adopting the model in such a context.

As suggested by participants, improving the S-CORT model's efficiency could be done by integrating different training options that favor self-learning through a blended approach of self-study and in-person training. The advanced distribution of a hard and soft copy of the learning resources materials could be a first step especially in areas with limited digital coverage. Notwithstanding, the use of mobile phones and other digital health technologies in a growing number of fragile and humanitarian settings with reliable mobile phone ownership and connectivity offers a promising platform for adapting the S-CORT materials for blended learning [32].

\section{Conclusion}


Accessing family planning services and LARC methods has been a significant shortcoming in humanitarian settings. The S-CORT model on LARCs offers a rapid competency-based refresher training for service providers needing an update and could, therefore, contribute to the full implementation of the MISP and allow crisis-affected individuals to have broader SRH choices. Such a capacity-development approach could be valuable not only for emergency response but in contexts engaged in disaster preparedness planning.

\section{Abbreviations}

DRC: Democratic Republic of Congo; MISP: Minimal Initial Service Package for Sexual and Reproductive Health in Humanitarian Settings; S-CORT: Sexual and Reproductive Health Clinical Outreach Refresh Training; SRH: Sexual and Reproductive Health.

\section{Declarations}

\section{Ethics approval and consent to participate}

The workshop evaluations were part of planned program monitoring, which was not designed to develop and contribute to generalizable knowledge and therefore did not constitute research and require ethical approval (Royse D, Thyer BA, Padgett DK, 2009) -this was confirmed by our submission to the Western Institutional Review Board (No. 2633824-44635729). No patient participated in the evaluation.

\section{Consent for publication}

Not applicable.

\section{Availability of data and materials}

Data is available upon reasonable request from the corresponding author.

\section{Competing interests}

The authors declare they have no competing interests. The opinions expressed are those of the authors and do not necessarily reflect the views of the funding agencies.

\section{Funding}

This project was funded by the Government of the Netherlands. 


\section{Authors' contributions}

NTT, AG, and AK conceived the evaluation tools. SZ, JG, AKMA, and BKS contributed to roll-out the incountry pilots with the support of AK, KG, and AG. SZ, JG, AKMA, and BKS collected the data with the support of KG, AK, and AG. NTT and AG conducted the analysis. NTT drafted the initial manuscript. SZ, $A G, A K$, and $K G$ contributed to the following drafts. All authors contributed to the final manuscript revision and have approved the final version.

\section{Acknowledgments}

We thank all the workshop participants for their time and contributions.

\section{Competing interests}

None declared.

\section{References}

1. Guttmacher Institute: Adding It Up - Investing in Sexual and Reproductive Health in Low- and MiddleIncome Countries. Fact Sheet. July 2020. Available at: https://www.guttmacher.org/sites/default/files/factsheet/adding-it-up-investing-in-sexualreproductive-health.pdf. Accessed on 17 Dec 2020.

2. Sully E, Biddlecom A, Darroch J, Ashford L, Lince-Deroche N, Riley T: Adding It Up: Investing in Sexual and Reproductive Health, 2020. New York, NY: Guttmacher Institute.

3. Ganatra B, Gerdts C, Rossier C, Johnson Jr BR, Tunçalp Ö, Assifi A, Sedgh G, Singh S, Bankole A, Popinchalk A: Global, regional, and subregional classification of abortions by safety, 2010-14: estimates from a Bayesian hierarchical model. The Lancet 2017, 390(10110):2372-2381.

4. Say L, Chou D, Gemmill A, Tunçalp Ö, Moller A-B, Daniels J, Gülmezoglu AM, Temmerman M, Alkema L: Global causes of maternal death: a WHO systematic analysis. The Lancet Global Health 2014, 2(6):e323-e333.

5. McGinn T, Bhabha J, Garfield R, Johnson K, Luchsinger G, Oddy L, Onyango MA, Shetir S, Searle L: Shelter from the storm: a transformative agenda for women and girls in a crisis-prone world. Columbia: United Nations Population Fund 2015.

6. Krause S, Williams H, Onyango MA, Sami S, Doedens W, Giga N, Stone E, Tomczyk B: Reproductive health services for Syrian refugees in Zaatri camp and Irbid City, Hashemite Kingdom of Jordan: an evaluation of the minimum initial services package. Conflict and health 2015, 9(1):S4.

7. Casey SE, Chynoweth SK, Cornier N, Gallagher MC, Wheeler EE: Progress and gaps in reproductive health services in three humanitarian settings: mixed-methods case studies. Conflict and health 2015, 9(1):S3. 
8. Casey SE, Cannon A, Mushagalusa Balikubirhi B, Muyisa J-B, Amsalu R, Tsolka M: Twelve-month contraceptive continuation among women initiating short-and long-acting reversible contraceptives in North Kivu, Democratic Republic of the Congo. PloS one 2017, 12(9):e0182744.

9. Singh NS, Aryasinghe S, Smith J, Khosla R, Say L, Blanchet K: A long way to go: a systematic review to assess the utilisation of sexual and reproductive health services during humanitarian crises. $B M J$ Glob Health 2018, 3(2):e000682.

10. Gallagher M, Morris C, Aldogani M, Eldred C, Shire AH, Monaghan E, Ashraf S, Meyers J, Amsalu R: Postabortion Care in Humanitarian Emergencies: Improving Treatment and Reducing Recurrence. Global Health: Science and Practice 2019, 7(Supplement 2):S231-S246.

11. Tanabe M, Schaus K, Rastogi S, Krause SK, Patel P: Tracking humanitarian funding for reproductive health: a systematic analysis of health and protection proposals from 2002-2013. Conflict and health 2015, 9(S1):S2.

12. Blanchet K, Sistenich V, Ramesh A, Frison S, Warren E, Hossain M, Knight A, Lewis C, Smith J, Woodward A: An evidence review of research on health interventions in humanitarian crises. London: London School of Hygiene \& Tropical Medicine 2013.

13. Sphere Project: Sexual and reproductive health in Sphere Handbook - Humanitarian charter and minimum standards in humanitarian response. Geneva: Sphere Project; 2018.

https://handbook.spherestandards.org/en/sphere/\#ch009_004.

14. Inter-Agency Working Group on Reproductive Health in Crises (IAWG): Minimum Initial Service Package (MISP). New York. ; 2018. https://iawgfieldmanual.com/manual/misp. Accessed 15 May 2020.

15. Foster AM, Evans DP, Garcia M, Knaster S, Krause S, McGinn T, Rich S, Shah M, Tappis H, Wheeler E: The 2018 Inter-agency field manual on reproductive health in humanitarian settings: revising the global standards. Reproductive Health Matters 2017, 25(51):18-24.

16. Women's Refugee Commission: A Clear Case for Need and Demand: Accessing Contraceptive Services for Rohingya Women and Girls in Cox's Bazar - Case Study. New York; June 2019. https://www.womensrefugeecommission.org/research-resources/contraceptive-service-delivery-inthe-refugee-camps-of-cox-s-bazar-bangladesh/. Accessed 29 Dec 2020

17. Women's Refugee Commission: Gap between Supply and Demand for Contraceptive Services in Northeast Nigeria - Case Study. New York; 2020.

https://www.womensrefugeecommission.org/research-resources/contraceptive-services-gapnigeria/. Accessed 29 Dec 2020.

18. Women's Refugee Commission: Opportunities and Challenges for Contraceptive Service Delivery in Cyclone Idai-Affected Areas of Mozambique - Case Study. New York; 2020. https://www.womensrefugeecommission.org/research-resources/opportunities-challenges-forcontraceptive-service-delivery-in-cyclone-idai-affected-areas-mozambique/. Accessed 29 Dec 2020.

19. Tran NT, Harker K, Yameogo WME, Kouanda S, Millogo T, Menna ED, Lohani JR, Maharjan O, Beda SJ, Odinga EA: Clinical outreach refresher trainings in crisis settings (S-CORT): clinical management of 
sexual violence survivors and manual vacuum aspiration in Burkina Faso, Nepal, and South Sudan. Reproductive health matters 2017, 25(51):103-113.

20. Giri S, Risnes K, Uleberg O, Rogne T, Shrestha SK, Nygaard ØP, Koju R, Solligård E: Impact of 2015 earthquakes on a local hospital in Nepal: A prospective hospital-based study. PloS one 2018, 13(2): $\mathrm{e} 0192076$.

21. Uprety M, Dugar S, Gautam D, Budimir M, Parajuli B, Kharbuja R: The Genesis of August 2017 Nepal Floods. AGUFM 2017, 2017:NH51D-05.

22. World Health Organization: Maternal mortality: Levels and trends 2000-2017; Estimates from WHO, UNICEF, UNFPA, World Bank Group and the United Nations Population Division. Geneva: World Health Organization; 2017. https://www.who.int/reproductivehealth/publications/maternal-mortality-20002017/en/.

23. Prakash D, Pandey J, Bietsch K: Unmet Need for Family Planning and Fertility in Nepal: Levels, Trends, and Determinants. DHS Further Analysis Reports No. 119. Rockville, Maryland, USA: ICF; 2019. https://dhsprogram.com/pubs/pdf/FA119/FA119.pdf. Accessed Jan 2020.

24. Ministry of Health of Nepal: Nepal Health Facility Suvery 2015. Kathmandu, Nepal; 2017. https://dhsprogram.com/pubs/pdf/spa24/spa24.pdf. Accessed 11 Jan 2021.

25. USAID, World Health Organisation, United Nations Population Fund: Training Resource Package for Family Planning; https://www.fptraining.org; 2021.

26. World Health Organization, USAID: Family planning: a global handbook for providers. https://www.fphandbook.org/. 2018.

27. Royse D, Thyer BA, Padgett DK: Program evaluation: An introduction: Cengage Learning; 2009.

28. Tran NT, Greer A, Dah T, Malilo B, Kakule B, Morisho TF, Asifiwe DK, Musa H, Simon J, Meyers J: Strengthening healthcare providers' capacity for safe abortion and post-abortion care services in humanitarian settings: lessons learned from the clinical outreach refresher training model (S-CORT) in Uganda, Nigeria, and the Democratic Republic of Congo. 2020.

29. Sharma DC: Nepal earthquake exposes gaps in disaster preparedness. The Lancet 2015, 385(9980):1819-1820.

30. Chaudhary P, Vallese G, Thapa M, Alvarez VB, Pradhan LM, Bajracharya K, Sekine K, Adhikari S, Samuel R, Goyet S: Humanitarian response to reproductive and sexual health needs in a disaster: the Nepal earthquake 2015 case study. Reproductive Health Matters 2017, 25(51):25-39.

31. Myers A, Sami S, Onyango MA, Karki H, Anggraini R, Krause S: Facilitators and barriers in implementing the Minimum Initial Services Package (MISP) for reproductive health in Nepal postearthquake. Conflict and health 2018, 12(1):1-9.

32. International Telecommunication Union: Yearbook of Statistics - Telecommunication/ICT Indicators 2009-2018. In., 2019 edn. Geneva: ITU; 2019. 